SUBMitTed to Astrophysical Journal Letters

Preprint typeset using LTEX style emulateapj v. 6/22/04

\title{
A CURIOUS NEW MILKY WAY SATELLITE IN URSA MAJOR ${ }^{0}$
}

D. B. Zucker ${ }^{1}$, V. Belokurov ${ }^{1}$, N. W. Evans ${ }^{1}$, J. T. Kleyna ${ }^{2}$, M. J. Irwin ${ }^{1}$, M. I. Wilkinson ${ }^{1}$, M. Fellhauer ${ }^{1}$, D. M. Bramich ${ }^{1}$, G. Gilmore ${ }^{1}$, H. J. Newberg ${ }^{3}$, B. Yanny ${ }^{4}$, J. A. Smith ${ }^{5,6}$, P. C. Hewett ${ }^{1}$, E. F. Bell ${ }^{7}$, H.-W. Rix ${ }^{7}$, O. Y. Gnedin ${ }^{8}$, S. Vidrih ${ }^{1}$, R. F. G. Wyse ${ }^{9}$, B. Willmai ${ }^{10}$, E. K. Grebel ${ }^{11}$, D. P. Schneider ${ }^{12}$, T. C. Beers ${ }^{13}$, A. Y. KNiazev ${ }^{7}, 14$ J. C. Barentine ${ }^{15}$, H. Brewington ${ }^{15}$, J. BrinkmanN ${ }^{15}$, M. HarvaneK ${ }^{15}$, S. J. KLEINMAN $^{16}$, J. KrZeSinski ${ }^{15,17}$, D. LONG ${ }^{15}$, A. NitTA ${ }^{18}$, S. A. SNEDDEN ${ }^{15}$

SUBMITTED To Astrophysical Journal Letters

\begin{abstract}
In this Letter, we study a localized stellar overdensity in the constellation of Ursa Major, first identified in Sloan Digital Sky Survey (SDSS) data and subsequently followed up with Subaru imaging. Its color-magnitude diagram (CMD) shows a well-defined sub-giant branch, main sequence and turn-off, from which we estimate a distance of $\sim 30 \mathrm{kpc}$ and a projected size of $\sim 250 \times 125 \mathrm{pc}$. The CMD suggests a composite population with some range in metallicity and/or age. Based on its extent and stellar population, we argue that this is a previously unknown satellite galaxy of the Milky Way, hereby named Ursa Major II (UMa II) after its constellation. Using SDSS data, we find an absolute magnitude of $M_{V} \sim-3.8$, which would make it the faintest known satellite galaxy. UMa II's isophotes are irregular and distorted with evidence for multiple concentrations; this suggests that the satellite is in the process of disruption.
\end{abstract}

Subject headings: galaxies: dwarf — galaxies: individual (Ursa Major II) — Local Group

\section{INTRODUCTION}

Numerical simulations in the hierarchical cold dark matter paradigm of galaxy formation generally predict 1 to 2 orders of magnitude more satellite halos in the present day Local Group than the number of dwarf galaxies thus far observed (e.g., Moore et al. 1999; Klvpin et al. 1999; Benson et al. 2002). Numerous solutions have been proposed for this "missing satellite" problem. For example, star formation may be inhibited in low-mass systems (e.g., Bullock et al. 2001; Somerville 2002), or the known satellites may represent a higher mass regime of the satellite initial mass function (e.g.,

${ }^{1}$ Institute of Astronomy, University of Cambridge, Madingley Road, Cambridge CB3 0HA, UK;zucker, vasily, nwe@ast.cam.ac.uk

${ }^{2}$ Institute for Astronomy, University of Hawaii, 2680 Woodlawn Drive, Honolulu, HI 96822

${ }^{3}$ Rensselaer Polytechnic Institute, Troy, NY 12180

${ }^{4}$ Fermi National Accelerator Laboratory, P.O. Box 500, Batavia, IL 60510

${ }^{5}$ Los Alamos National Laboratory, ISR-4, MS D448, Los Alamos, NM 87545

6 Department of Physics and Astronomy, Austin Peay State University, P.O. Box 4608, Clarksville, TN 37040

${ }^{7}$ Max Planck Institute for Astronomy, Königstuhl 17, 69117 Heidelberg, Germany

${ }^{8}$ Department of Astronomy, Ohio State University, 140 West 18th Avenue, Columbus, OH 43210

${ }^{9}$ The Johns Hopkins University, 3701 San Martin Drive, Baltimore, MD 21218

${ }_{10}$ Center for Cosmology and Particle Physics, Department of Physics, New York University, 4 Washington Place, New York, NY 10003

11 Astronomical Institute of the University of Basel, Department of Physics and Astronomy, Venusstrasse 7, CH-4102 Binningen, Switzerland

12 Department of Astronomy and Astrophysics, Pennsylvania State University, 525 Davey Laboratory, University Park, PA 16802

${ }^{13}$ Department of Physics and Astronomy, Michigan State University, East Lansing, MI 48824

${ }^{14}$ South African Astronomical Observatory, PO Box 9, Observatory 7935, Cape Town, South Africa

${ }^{15}$ Apache Point Observatory, P.O. Box 59, Sunspot, NM 88349

${ }^{16}$ Subaru Telescope, 650 N. A'ohoku Place, Hilo, HI 96720

17 Mt. Suhora Observatory, Cracow Pedagogical University, ul. Podchorazych 2, 30-084 Cracow, Poland

${ }^{18}$ Gemini Observatory, 670 N. A'ohoku Place, Hilo, HI 96720

${ }^{0}$ Based in part on data collected at Subaru Telescope, which is operated by the National Astronomical Observatory of Japan.
TABLE 1

Properties of the Ursa Major II Dwarf

\begin{tabular}{|c|c|}
\hline \multicolumn{2}{|l|}{ Parameter $^{\mathrm{a}}$} \\
\hline Coordinates (J2000) & $08: 51: 30+63: 07: 48$ \\
\hline Coordinates (Galactic) & $\ell=152.5^{\circ}, b=37.4^{\circ}$ \\
\hline Position Angle & $95^{\circ}$ \\
\hline Ellipticity & 0.5 \\
\hline Central Extinction, $A_{V}$ & 0.29 \\
\hline $\mathrm{V}_{\text {tot }}$ & $14^{\mathrm{m}} \cdot 3 \pm 0.5$ \\
\hline$(\mathrm{m}-\mathrm{M})_{0}$ & $17.5 \pm 0.3$ \\
\hline $\mathrm{M}_{\mathrm{tot}, \mathrm{V}}$ & $-3 \cdot 8 \pm 0.6$ \\
\hline${ }^{\mathrm{a}}$ Integrated magnitudes & are corrected for the \\
\hline $\begin{array}{l}\text { Galactic foreground } \\
\text { Schlegel et al. (1998) }\end{array}$ & dening reported by \\
\hline
\end{tabular}

\section{Stoehr et al. 2002; Kravtsov et al. 2004).}

However, it has become increasingly clear over the last two years that the census of Local Group satellites is seriously incomplete. Data from the Sloan Digital Sky Survey (SDSS; York et al. 2000) have revealed five new nearby dwarf spheroidals (dSphs) in quick succession: Andromeda IX (Zucker et al. 2004), Ursa Major (Willman et al. 2005a), Andromeda $X$ (Zucker et al. 2006b), Canes Venatici (Zucker et al. 2006a) and Boötes (Belokurov et al. 2006b). All five galaxies were detected as stellar overdensities. The purpose of this Letter is to study another prominent stellar overdensity in SDSS Data Release 4 (Adelman-McCarthy et al. 2006). Grillmair (2006) independently called attention to it, stating that it may be a "new globular cluster or dwarf spheroidal". Here we provide evidence from SDSS and subsequent deeper Subaru imaging for its interpretation as a dwarf spheroidal galaxy, the thirteenth around the Milky Way, with the proposed name Ursa Major II (UMa II).

\section{OBSERVATIONS AND DATA ANALYSIS}

The SDSS is an imaging and spectroscopic survey, with imaging data taken in five photometric bands $(u, g$, $r, i$ and $z$; Fukugita et al. 1996; Gunn et al. 1998, 2006; 

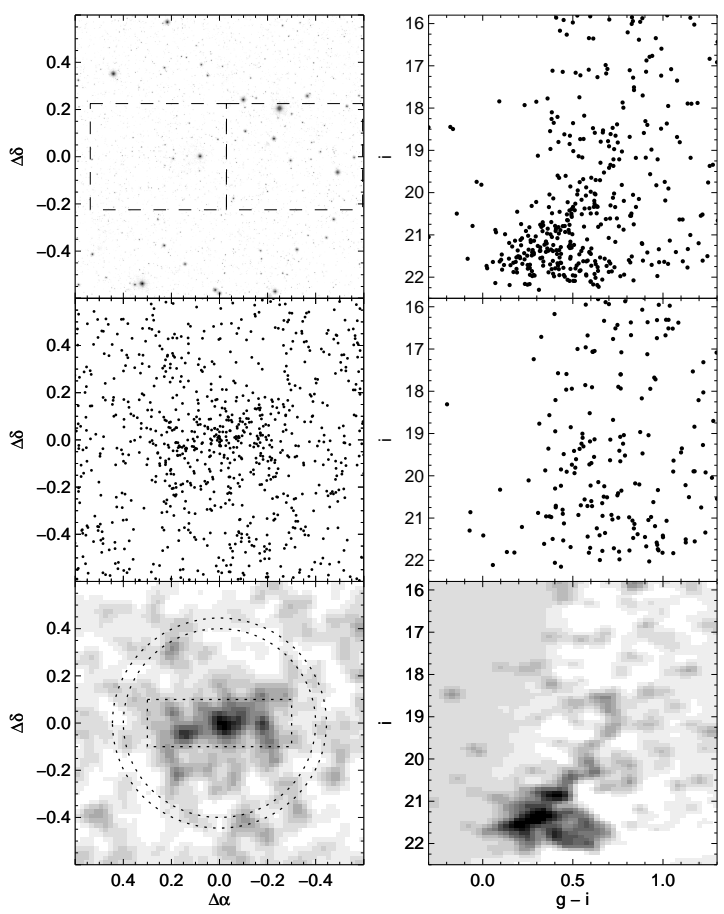

FIG. 1.- The UMa II Dwarf as seen by SDSS: Upper left: Combined SDSS $g, r, i$ images of a $1.2^{\circ} \times 1.2^{\circ}$ field centered on the overdensity (J2000 08:51:30 $+63: 07: 48) . \Delta \alpha$ and $\Delta \delta$ are the relative offsets in right ascension and declination, measured in degrees of arc. The dashed lines indicate the two pointings observed with Subaru (see §2). Middle left: The spatial distribution of all blue objects $(g-i<0.5)$ classified as stars in the same area. Lower left: Binned spatial density of all blue stellar objects, together with a dotted box that covers most of the object and a dotted annulus used to define the background. Upper right: CMD of all stellar objects within the dotted box; note the clear main sequence turn-off and subgiant branch, along with hints of horizontal and red giant branches, even without removal of field contamination. Middle right: Control CMD of field stars from the dotted annulus. Lower right: A color-magnitude density plot (Hess diagram), showing the CMD of the box minus the control CMD, normalized to the number of stars in each CMD. All photometric data were corrected for Galactic foreground extinction using Schlegel et al. (1998).

Hogg et al. 2001; Adelman-McCarthy et al. 2006) and automatically processed through photometric and astrometric pipelines (Lupton, Gunn, \& Szalav 1999; Stoughton et al. 2002; Smith et al. 2002; Pier et al. 2003; Ivezić et al. 2004). As part of our systematic analysis of SDSS data around the north Galactic pole (see, e.g., Belokurov et al. 2006a), we identified a stellar overdensity in the constellation Ursa Major.

Figure 11 shows a set of panels derived from the SDSS public data. A combined $g, r, i$ grayscale image centered on the stellar overdensity (upper left) reveals no obvious object, However, by selecting only the objects classified by the SDSS pipeline as blue stars $(g-i<0.5)$, a flattened stellar overdensity is readily visible in the photometric data (middle and bottom left panels). A color-magnitude diagram (CMD) of all stars in the central region reveals a clear main sequence turn-off and sub-giant branch, as well as what appear to be a red clump and sparse horizontal and red giant branches (right panels). The CMD bears some resemblance to those of intermediate-metallicity globular clusters, but the satellite's seemingly irregular and broken morphology with distinct blobs and sub-clumps leaves its precise nature open to question. It is not even clear that it is a single object.

Accordingly, we obtained deeper follow-up observations with the Suprime-Cam mosaic imager (Mivazaki et al. 2002) on the Subaru telescope (Ive et al. 2004). Data were gathered on 2006 May 26 (UT), using two pointings to cover the stellar
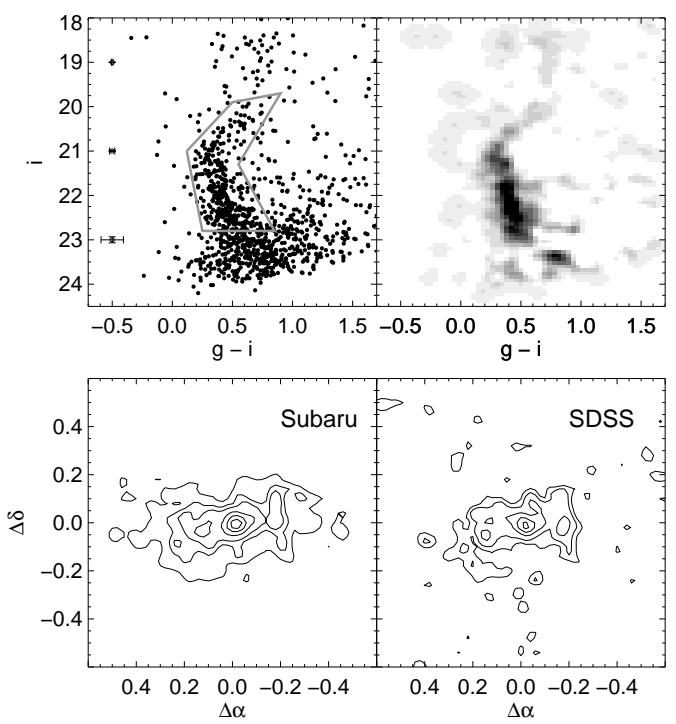

FIg. 2.- The UMa II Dwarf as seen by Subaru: Upper left: CMD of the central region of UMa II (see dashed box in the upper right panel of Figure11, constructed with Subaru $g, i$ data. The solid gray line graphically indicates the color-magnitude selection criteria used to construct the contour plots in the lower panels. The error bars on the left show the typical photometric errors at the $i$-band magnitude indicated. Upper right: A color-magnitude density plot (Hess diagram), showing the CMD of the box minus a control-field CMD, normalized to the number of stars in each CMD. Lower left: Isodensity contours of the stars selected from the Subaru data by the gray box in the upper left panel. The plotted contour levels are 1,2,3, 5, 7 and $9 \sigma$ above the background level. $\Delta \alpha$ and $\Delta \delta$ are measured in degrees of arc. Lower right: Isodensity contours using SDSS data for comparison, with levels of 2, 3, 5 , 7 and $9 \sigma$ above the background plotted. Note that the three blobs appear in both panels.

overdensity (upper left panel of Figure 1). Each pointing was observed in $g^{\prime}$ and $i^{\prime}$ bands (for ease of calibration with SDSS data) in a 3-exposure dither to cover the gaps between CCDs. Each exposure was 240s, for 12 minutes of exposure time in each band. Unfortunately, several exposures of the western pointing were affected by problems with vignetting and tracking, so that only single $g^{\prime}$ and $i^{\prime}$ exposures of this area were usable. The data were processed using a general purpose pipeline modified for Subaru reductions. Images were debiased and trimmed, and then flatfielded and gain-corrected to a common internal system using clipped median stacks of nightly twilight flats. Aperture photometry from these processed images was then bootstrap calibrated onto the SDSS photometric system.

The upper left panel of Figure 2 shows a deep CMD derived from our Subaru imaging. A densely populated upper main sequence and sub-giant branch are now clearly discernible, though with only a truncated red giant branch and possible horizontal branch because of saturation in the Subaru data brighter than $i \sim 18$. The upper right panel of Figure 2] shows a background-subtracted Hess diagram of the object. The solid gray line that wraps around the object's main sequence and sub-giant branch in the upper left panel is used to select members. The density contours derived from the spatial distribution of these stars are shown in the lower left panel. The central parts of the object break up into three distinct clumps. These are also visible in the density contours derived from the SDSS data, using the same color-magnitude selection, giving additional confidence that they are not merely data artifacts.

\section{PROPERTIES OF UMA II}

The upper left panel of Figure 3 shows a composite CMD of the central parts of the object with the bright stars taken 

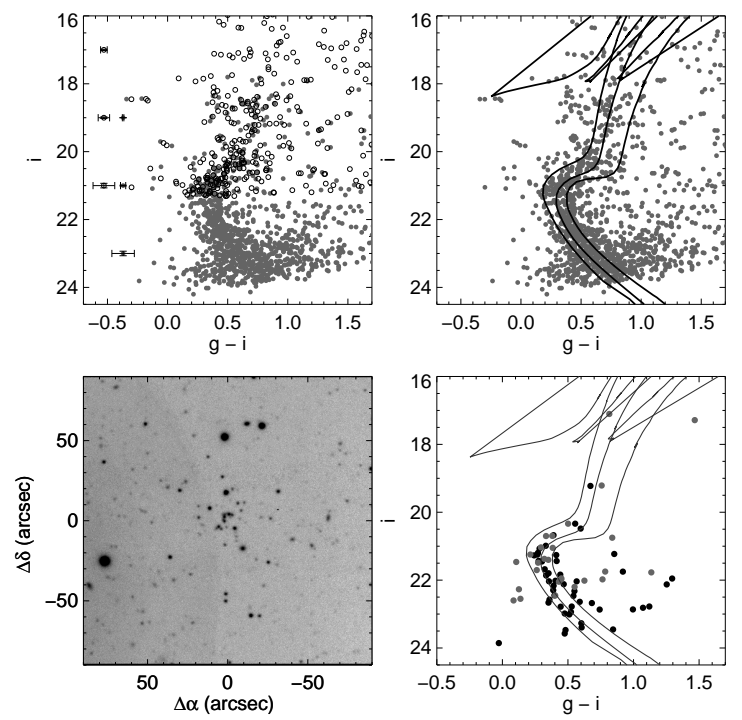

FIG. 3.- Upper left: Composite CMD of the central region of UMa II (dashed box in the upper right panel of Figure 1, with photometry of both SDSS and Subaru stars plotted (black circles and gray dots, respectively). Duplicate detections (i.e., detections of the same star in both sets of photometric data) have not been removed. The error bars on the left show the typical photometric errors for each dataset at the $i$-band magnitude indicated. Upper right: The same composite CMD, with all stars shown as gray dots, and Padova isochrones Girardi et al. 2004) overplotted for (left to right) $[\mathrm{Fe} / \mathrm{H}]=-2.3 / 12 \mathrm{Gyr},[\mathrm{Fe} / \mathrm{H}]=-1.3 / 12 \mathrm{Gyr}$ and $[\mathrm{Fe} / \mathrm{H}]=-0.7 / 10$ Gyr, shifted to a distance modulus of 17.5. Lower left: Subaru $g$-band image of the apparent central cluster of UMa II. The image spans $3^{\prime} \times 3^{\prime}$. The curved shadow to the left is scattered light from a nearby bright star. Lower right: Composite CMD of the central cluster region shown in the lower left panel, with SDSS and Subaru photometry plotted as gray and black dots, respectively. The three isochrones from the upper right panel are also overplotted; the middle isochrone $([\mathrm{Fe} / \mathrm{H}]=-1.3 / 12 \mathrm{Gyr})$ appears to be a reasonably good fit to the data, although even in this small region the main sequence is broader than might be expected from simple photometric errors (see upper left panel).

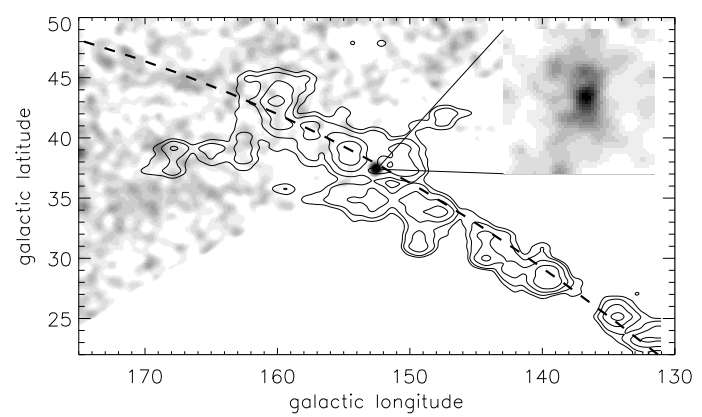

FIg. 4.- The locations of UMa II and Complex A, together with the great circle of the Orphan Stream. The distance estimate to the Orphan Stream is comparable to that of UMa II, but Complex A is believed to lie much closer. The gray scale shows the density of SDSS stars satisfying $g-r<0.4$ and $20<$ $r<22.5$. The inset image is a blow-up of the area immediately around UMa II, showing its long axis almost aligned with constant Galactic longitude. The column density contours for Complex A are taken from Wakker (2001), while the great circle of the Orphan Stream is from Belokurov et al. (2006c).

from SDSS and the faint ones from Subaru. The width in the upper main sequence far exceeds the observational errors and the expected range in foreground extinction $(\Delta E(g-i) \sim 0.1$; Schlegel et al. 1998) and may be caused by a number of factors. First, there is nebulosity in the field of our $g^{\prime}$ band Subaru images, suggesting that there may be patchy reddening unresolved on the scale of Schlegel et al.'s (1998) maps. Second, the spread could be caused by depth along the line of sight, although the main sequence is nearly vertical near the turn-off and thus a distance spread alone would not reproduce its observed width. Finally, it could be caused by a mix of stellar populations of different metallicity and age. This last hypothesis is illustrated in the upper right panel of Figure 3 by the overplotting of isochrones of different metallicities and ages from Girardi et al. (2004). The stellar population is not well-described by a single isochrone, but the data are consistent with a single distance, and an age/metallicity range. Judging from the isochrones, a reasonable conclusion is that the object is of intermediate metallicity and at least 10 Gyrs old. A Subaru $g^{\prime}$ band image of what appears to be a central cluster is shown in the lower left panel of Figure 3 The image is dominated by turn-off and sub-giant stars (lower right panel). Even in the small area of the central cluster, the main sequence appears to be broader than that of a single population.

Given the breadth of the main sequence and turn-off, it is difficult to determine a precise distance to the object. From the overlaid isochrones, we estimate a distance modulus of $(m-M)_{0} \sim 17.5 \pm 0.3$, corresponding to $\sim 30 \pm 5 \mathrm{kpc}$. At that distance, the angular extent of the object $\left(\sim 0.5^{\circ} \times 0.25^{\circ}\right)$ translates to a size of $\sim 250 \times 125 \mathrm{pc}$. Using the same method described in Belokurov et al. (2006b), we estimate its absolute magnitude as $M_{V} \sim-3.8 \pm 0.6$, a value consistent with the absence of a significant number of giant stars. Based on its size (which exceeds typical values for faint globular clusters), its broad CMD morphology (which argues against a single stellar population), and its extremely low surface brightness, we conclude that this is most likely a hitherto unknown $\mathrm{dSph}$ galaxy. As it is the second Milky Way dSph satellite to be discovered in this constellation, we follow convention in naming it Ursa Major II (UMa II).

\section{DISCUSSION}

At $M_{V} \sim-3.8$, UMa II would be the faintest dSph yet discovered. One might therefore wonder whether UMa II could instead be a large globular cluster with gross tidal distortions. In a globular cluster undergoing tidal disruption, the transverse size of the tail does not increase appreciably (Dehnen et al. 2004). Thus the diameter of the globular cluster would have to be $\sim 125 \mathrm{pc}$, larger than almost all known globulars. In addition, the CMD does not resemble that of a single stellar population, as in a typical globular cluster. If UMa II were a disrupted cluster, the progenitor would likely have had properties more extreme than the largest Milky Way globular, $\omega$ Centauri, itself widely believed to be the nucleus of a dSph (e.g., Majewski et al. 2000).

Yet, in its physical properties UMa II does resemble Willman 1 - a peculiar object also found with SDSS - which may be a tidally-disrupted globular cluster (Willman et al. 2005b, 2006). Willman 1's absolute magnitude and half-light radius, $M_{V} \sim-2.5$ and $r_{1 / 2} \sim 20 \mathrm{pc}$ (Willman et al. 2006), are at least a factor of $\sim 3$ fainter and smaller than the corresponding quantities for UMa II, $M_{V} \sim-3.8$ and $r_{1 / 2} \sim 50 \mathrm{pc}$ or $\sim 120 \mathrm{pc}$ (based on the minor axis or azimuthally averaged). In the $M_{V}$ vs. $r_{1 / 2}$ plane, UMa II would lie between Willman 1 and the recently discovered low-luminosity Milky Way dSph satellites Ursa Major I, Boötes and Canes Venatici. With digital surveys like SDSS we are thus probing a new regime of ultra-low surface brightness stellar structures, where - in the absence of kinematic data - the distinction between globular clusters and dwarf galaxies is no longer obvious.

If the thickness of the main sequence is indicative of episodic or extended star formation, then UMa II may once have been much more massive and subsequently suffered disruption. The isophotes of UMa II are even more distorted 
and irregular than those of the Ursa Minor (Palma et al. 2003) or Bootes dSphs (Belokurov et al. 2006b). In addition to the central cluster, there appear to be two density peaks at $\left(\Delta \alpha \sim 0.2^{\circ}, \Delta \delta \sim 0.0^{\circ}\right)$ and at $\left(\Delta \alpha \sim-0.2^{\circ}, \Delta \delta \sim-0.05^{\circ}\right)$. These may perhaps be just fragments of what was once a regular galaxy. If UMa II were unbound, the fragments would probably have been completely disrupted by now and would not be detected as a significant stellar overdensity.

But is UMa II gravitationally bound? We can estimate what mass-to-light ratio $(\mathrm{M} / \mathrm{L})$ would be required for it to be bound using the criterion for tidal disruption of a cluster of particles in a circular orbit: $3 M_{\mathrm{MW}} / D_{\mathrm{MW}}^{3}>M_{\mathrm{UMaII}} / R_{\mathrm{UMaII}}^{3}$, where $M_{\mathrm{MW}}$ and $M_{\mathrm{UMaII}}$ are the enclosed masses of the Milky Way and UMa II, $D_{\mathrm{MW}}$ is the distance of UMa II from the center of the Milky Way, and $R_{\mathrm{UMaII}}$ is the radius of UMa II. Assuming $M_{\mathrm{MW}} \sim 4 \times 10^{11} M_{\odot}, D_{\mathrm{MW}} \sim 36 \mathrm{kpc}$ (from a heliocentric distance of $\sim 30 \mathrm{kpc}), R_{\mathrm{UMaII}} \sim 100 \mathrm{pc}$, and $L_{\mathrm{UMaII}} \sim 3 \times 10^{3} L_{\odot}$ (based on $M_{V, U M a I I} \sim-3.8$ and $M_{V, \odot} \sim 4.85$ ), UMa II would require $\mathrm{M} / \mathrm{L} \sim 8$ to be marginally bound. The uncertainties in this estimate are substantial - just the approximations inherent in the formula could introduce errors of a factor of $\sim 4-$ but it does suggest that, if UMa II is gravitationally bound, its $\mathrm{M} / \mathrm{L}$ may be higher than that of a typical stellar population. In other words, the existence of UMa II as a presumably longlived, distinct object could imply a higher M/L than would be expected in a system without dark matter.

UMa II is found in a busy area of sky, as shown in Figure 4 UMa II lies on the great circle of the "Orphan Stream", a $50^{\circ}$ stellar stream discovered in SDSS data (Belokurov et al. 2006c; Grillmair 2006). The distance to the Orphan Stream is $\sim 30 \mathrm{kpc}$, comparable to UMa II. The great circle of the Orphan Stream includes a number of anomalous, young halo globular clusters, particularly Palomar 1 and Ruprecht 106. UMa II also lies close to the association of HI high velocity clouds known as Complex A (see e.g., Wakker 2001). Complex A has a distance bracket of 4.0 to $10.1 \mathrm{kpc}$ (Wakker et al. 1996). Although this is much closer than UMa II, nonetheless they may be associated if Complex A lies on a different orbital wrap of a mutual progenitor (Belokurov et al. 2006c). UMa II could thus be a surviving fragment of a larger progenitor.

\section{CONCLUSIONS}

We have identified a new companion to the Milky Way galaxy in the constellation Ursa Major. Based on its size, structure and stellar population, we argue that it is a new dwarf spheroidal galaxy and name it UMa II. It has a distance of $\sim 30 \mathrm{kpc}$ and an absolute magnitude of $M_{V} \sim-3.8$. Its colormagnitude diagram shows an upper main sequence, turn-off and sub-giant branch, as well as hints of red giant and horizontal branches. UMa II has a bright central concentration, together with two further clumps. The irregular nature of the object suggests that it may have undergone disruption.

This is the fourth Milky Way dSph discovered by SDSS in little over a year. Together with the earlier discoveries of Ursa Major I, Canes Venatici and Boötes, this underscores how incomplete our current census actually is. As SDSS covers only $\sim 1 / 4$ of the celestial sphere, crude scaling arguments would suggest that there are tens of missing Milky Way dSphs. If true, this would go some way toward resolving the missing satellite issue.

Funding for the SDSS and SDSS-II has been provided by the Alfred P. Sloan Foundation, the Participating Institutions, the National Science Foundation, the U.S. Department of Energy, the National Aeronautics and Space Administration, the Japanese Monbukagakusho, the Max Planck Society, and the Higher Education Funding Council for England.The SDSS Web Site is http://www.sdss.org/

The SDSS is managed by the Astrophysical Research Consortium for the Participating Institutions. The Participating Institutions are the American Museum of Natural History, Astrophysical Institute Potsdam, University of Basel, Cambridge University, Case Western Reserve University, University of Chicago, Drexel University, Fermilab, the Institute for Advanced Study, the Japan Participation Group, Johns Hopkins University, the Joint Institute for Nuclear Astrophysics, the Kavli Institute for Particle Astrophysics and Cosmology, the Korean Scientist Group, the Chinese Academy of Sciences (LAMOST), Los Alamos National Laboratory, the Max-Planck-Institute for Astronomy (MPIA), the MaxPlanck-Institute for Astrophysics (MPA), New Mexico State University, Ohio State University, University of Pittsburgh, University of Portsmouth, Princeton University, the United States Naval Observatory, and the University of Washington.

\section{REFERENCES}

Adelman-McCarthy, J. K., et al. 2006, ApJS, 162, 38

Belokurov, V. et al. 2006a, ApJ, 642, L137

Belokurov, V. et al. 2006b, ApJ, in press

Belokurov, V. et al. 2006c, ApJ, submitted

Benson, A. J., Frenk, C. S., Lacey, C. G., Baugh, C. M., \& Cole, S. 2002, MNRAS, 333, 177

Bullock, J. S., Kravtsov, A. V., \& Weinberg, D. H. 2001, ApJ, 548, 33

Dehnen, W., Odenkirchen, M., Grebel, E. K., \& Rix, H.-W. 2004, AJ, 127, 2753

Fukugita, M., Ichikawa, T., Gunn, J. E., Doi, M., Shimasaku, K., \& Schneider, D. P. 1996 , AJ, 111, 1748

Girardi, L., Grebel, E. K., Odenkirchen, M., and Chiosi, C. 2004, å, 422, 205

Grillmair, C. 2006, ApJ, in press ' astro-ph/0605396

Gunn, J.E. et al. 1998, AJ, 116, 3040

Gunn, J.E. et al. 2006, ApJ, 131, 2332

Hogg, D.W., Finkbeiner, D.P., Schlegel, D.J., Gunn, J.E. 2001, AJ, 122, 2129

Ivezić, Ž. et al., 2004, AN, 325, 583

Iye, M. et al., 2004, PASJ, 54, 833

Klypin, A., Kravtsov, A. V., Valenzuela, O., \& Prada, F. 1999, ApJ, 522, 82

Kravtsov, A., Gnedin, O., \& Klypin, A. 2004, ApJ, 609, 482

Lupton, R., Gunn, J., \& Szalay, A. 1999, AJ, 118, 1406

Majewski, S. R., Patterson, R. J., Dinescu, D. I., Johnson, W. Y., Ostheimer

J. C., Kunkel, W. E., \& Palma, C. 2000, Liege International Astrophysical Colloquia, 35, 619

Miyazaki, S., et al. 2002, PASJ, 54, 833
Moore, B., Governato, F., Lake, G., Quinn, T., Stadel, J., \& Tozzi, P. 1999, ApJ, 524, L19

Palma, C., Majewski, S. R., Siegel, M. H., Patterson, R. J., Ostheimer, J. C., \& Link, R. 2003, AJ, 125, 1352

Pier, J.R., Munn, J.A., Hindsley, R.B, Hennessy, G.S., Kent, S.M., Lupton, R.H., Ivezic, Z. 2003, AJ, 125, 1559

Schlegel, D. J., Finkbeiner, D. P., \& Davis, M. 1998, ApJ, 500, 525

Smith, J. A., et al. 2002, AJ, 123, 2121

Somerville, R. S. 2002, ApJ, 572, L23

Stoehr, F., White, S. D. M., Tormen, G., \& Springel, V. 2002, MNRAS, 335, L84

Stoughton, C. et al. 2002, AJ, 123, 485

York D.G., et al. 2000, AJ, 120, 1579

Wakker, B. 2001, ApJS, 136, 463

Wakker B., Howk C., Schwarz, U., van Woerden, H., Beers, T., Wilhelm, R., Kalberla, P., \& Danly, L. 1996, ApJ, 473, 834

Willman, B., et al. 2005a, ApJ, 626, L85

Willman, B., et al. 2005b, AJ, 129, 2692

Willman, B., et al. 2006, AJ, submitted (astro-ph/0603486

Zucker et al. 2004, ApJ, 612, L121

Zucker, D. B., et al. 2006a, ApJ, 643, L103

Zucker, D. B., et al. 2006b, ApJ, submitted /astro-ph/0601599 